\title{
Identification of Hysteresis Functions Using a Multiple Model Approach
}

\author{
Lyudmila Mihaylova*, Vincent Lampaert, Herman Bruyninckx, Jan Swevers \\ Katholieke Universiteit Leuven, Department of Mechanical Engineering \\ Celestijnenlaan 300 B, B-3001 Heverlee, Belgium \\ Tel.: 00321632 2533, E-mail: Lyudmila.Mihaylova@mech.kuleuven.ac.be
}

\begin{abstract}
This paper considers the identification of static hysteresis functions which describe phenomena in mechanical systems, piezoelectric actuators and materials. A solution based on a model with a parallel structure of elementary models (with switching) and the Interacting Multiple Model (IMM) approach is proposed. For each elementary model a separate IMM estimator is implemented. The estimated parameters represent a fusion of values from preset grids, weighted by the IMM mode probabilities. The estimated state of each elementary model is a fusion of the estimated states (from the separate Kalman filters) weighted by the IMM probabilities. The nonlinear identification problem is reduced to a linear one. Results from simulation experiments are presented.
\end{abstract}

Key words - nonlinear systems, mechatronics, multiplemodels estimation, system identification, hysteresis

\section{INTRODUCTION}

One of the phenomena where hysteresis appears is in friction. Friction is a nonlinear phenomenon occurring almost in all mechanical systems exhibiting hysteretic behavior. Different friction regimes can be distinguished - presliding region for movements over short distances (a few micrometers) in which the adhesive forces are dominant such that the friction force appears to be a hysteresis function of the displacement and a sliding region, for larger movements in which the friction force depends on the velocity. Different methods for modeling this phenomenon and identifying its characteristics are proposed, starting with classical descriptions between velocity and friction force (differential equations, static maps) which can not adequately describe the presliding region to the more complex LuGre [1,2] and Leuven [9] models. Those last two models are capable to describe both regions. The Leuven model [9] implements also the nonlocal memory hysteresis characteristic which is not the case for the LuGre model.

This paper considers only the presliding region. It investigates the identification of static hysteresis functions with nonlocal memory, i.e. functions for which

* On leave from the Bulgarian Academy of Sciences the future output value depends both on the current output value, and past extremums of the input. The term static indicates that the speed of the input variations has no influence on the shape of the hysteresis curve. The identification is performed by a multiple model (MM) approach (with the Interacting Multiple Model (IMM) estimator). The MM approach has recently proven to be powerful to solve problems with uncertainties (structural and parametric), abrupt changes in the system behavior, in decomposing a complex problem into simpler subproblems in various areas as target tracking [6], fault detection [8, 10] and identification [6, 7]. Using this approach the hysteresis function, characterized by a strong nonlinearity (switching function), can be decoupled into a set of linear functions yielding a linear description of the nonlinear phenomenon. The IMM estimator uses the model derived in [4, 5] which describes the hysteresis as a parallel structure of elementary models. The developed method can be used to identify each hysteresis function.

\section{PROBLEM FORMULATION}

The hysteresis behaviour can be described as a parallel connection (Fig.1) of elementary models [3, 5] with unknown parameters.

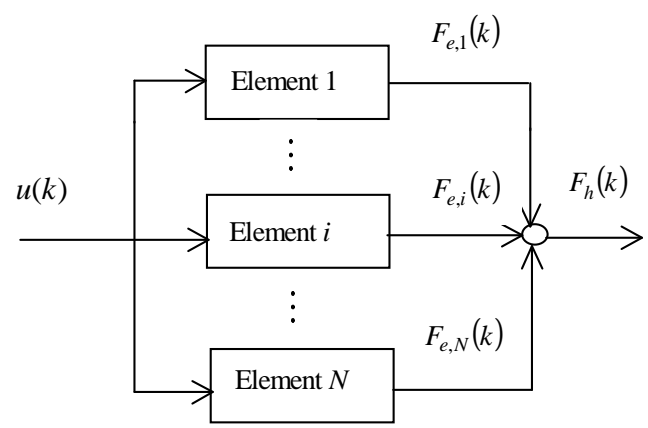

Fig. 1. Model of the hysteresis force based on parallel connection of elementary models

Each elementary model has one common displacement input $u(k)$ and one output force $F_{e, i}(k)$ (unmeasurable). The hysteresis force $F_{h}(k)$ corresponds to the sum 


$$
F_{h}(k)=\sum_{i=1}^{N} F_{e, i}(k)
$$

of the outputs $F_{e, i}(k)$ of the elementary models. Each elementary model has its own state $\zeta_{i}(k)$ and is characterized by two parameters: its maximum force $W_{i}$ (i.e. $W_{i} \in\left[0, F_{\max }\right]$ ) and the spring constant $K_{i}$.

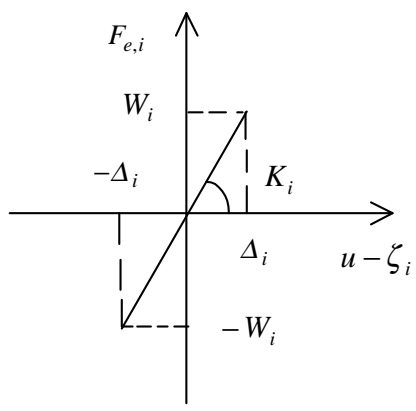

Fig. 2. The characteristic of the elementary model $i$

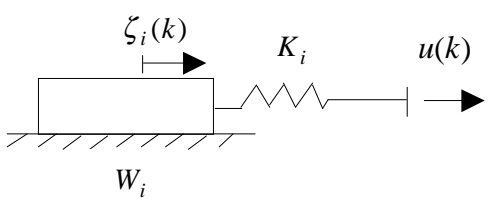

Fig. 3. Physical interpretation of the elementary model $i$

An elementary model relates the relative displacement $u(k)-\zeta_{i}(k)$ to the force $F_{e, i}(k)$. The relationship is shown in Fig. 2 and can be described by the equations:

- $\quad$ case 1 :

$$
\text { for } f_{i}(k) \in\left[-W_{i}, W_{i}\right]:\left\{\begin{array}{l}
\zeta_{i}(k+1)=\zeta_{i}(k) \\
F_{e, i}(k)=f_{i}(k)
\end{array},\right.
$$

- $\quad$ case 2:

$$
\text { for } f_{i}(k)>W_{i}:\left\{\begin{array}{l}
\zeta_{i}(k+1)=\zeta_{i}(k)+\frac{f_{i}(k)-W_{i}}{K_{i}} \\
F_{e, i}(k)=W_{i}
\end{array},\right.
$$

- $\quad$ case 3:

$$
\text { for } f_{i}(k)<-W_{i}:\left\{\begin{array}{l}
\zeta_{i}(k+1)=\zeta_{i}(k)+\frac{f_{i}(k)+W_{i}}{K_{i}} \\
F_{e, i}(k)=-W_{i}
\end{array},\right.
$$

where $f_{i}(k)=K_{i}\left[u(k)-\zeta_{i}(k)\right]$ is the switching parameter. Each elementary model can be considered as an elasto-slide element consisting of a massless block subject to a Coulomb friction connected to a massless linear spring (See Fig. 3). The parameter $W_{i}$ represents the Coulomb friction and the coefficient $K_{i}$ characterizes the spring constant. Another characteristic parameter of the elementary model which can be used is $\Delta_{i}=W_{i} / K_{i}, i=1,2, \ldots, N$, corresponding to the defor- mation of the spring when the spring force equals the Coulomb friction force. The deformation of the spring is the difference of the input displacement $u(k)$ and the position $\zeta_{i}(k)$ of the element. When the spring force is larger than the Coulomb friction force, the element begins to slide. Each block of an elementary model can remain in the same position called sticking (case 1), or can undergo a change of the position, called slipping (case 2 and 3). The parallel connection of the different elasto-slide elementary models (Fig.1) form the global static hysteresis model.

On the basis of (2)-(4), the following state-space description can be obtained

$$
\begin{aligned}
& x_{i}(k+1)=F_{i} x_{i}(k)+G_{i} u(k)+G_{\eta, i} \eta_{i}(k), \\
& y_{i}(k)=C_{i} x_{i}(k)+D_{i} u(k)+\xi_{i}(k),
\end{aligned}
$$

where the state $x_{i}(k)=\zeta_{i}(k)$ is the position of the $i-t h$ element, $u(k)$ is the input signal, $y_{i}(k)=F_{e, i}(k)$ is the output of the element. The respective model parameters are:

- case 1: for $f_{i}(k) \in\left[-W_{i}, W_{i}\right]$

$$
F_{i}=1, G_{i}=0, C_{i}=-K_{i}, D_{i}=K_{i} \text {; }
$$

- case 2: for $f_{i}(k)>W_{i}$

$$
\begin{aligned}
& x_{i}(k)=-W_{i} / K_{i}, F_{i}=1, G_{i}=1, \\
& C_{i}=-K_{i}, D_{i}=0 ;
\end{aligned}
$$

- case 3: for $f_{i}(k)<-W_{i}$

$$
\begin{aligned}
& x_{i}(k)=W_{i} / K_{i}, \\
& F_{i}=1, G_{i}=1, C_{i}=-K_{i}, D_{i}=0 .
\end{aligned}
$$

$\eta_{i}(k)$ and $\xi_{i}(k)$ are process and measurement noises, mutually uncorrelated, Gaussian, zero-mean, with covariances $Q_{i}$ and $R_{i} . G_{\eta, i}$ allows to change the influence of the process noise $\eta_{i}$, reflecting model and discretization errors due to the replacement of $\Delta_{i}$ and $K_{i}$ with grid values from uncertainty domains. There are $2 N$ parameters to identify, namely, $W_{i}$ and $K_{i}$ ( $i=$ $1,2, \ldots, N$ is the number of the elementary models used).

The connected in parallel $N$ elementary models form an augmented single-input single-output model

$$
\begin{aligned}
& \tilde{x}(k+1)=\tilde{F} \tilde{x}(k)+\tilde{G} u(k)+\tilde{G}_{\eta} \eta(k), \\
& y(k)=\tilde{C} x(k)+\tilde{D} u(k)+\xi(k),
\end{aligned}
$$

where $\tilde{x}(k+1)=\left[\zeta_{1}(k+1), \ldots, \zeta_{N}(k+1)\right]^{T}, y(k)=F_{h}(k)$

$$
\begin{gathered}
\tilde{F}=I_{N}, \tilde{G}=\left[G_{1}, \ldots, G_{N}\right]^{T}, \tilde{G}_{\eta}=\left[G_{\eta, 1}, \ldots, G_{\eta, N}\right]^{T}, \\
\tilde{C}=\left[-K_{1}, \ldots,-K_{N}\right], \tilde{D}=\left[D_{1}, \ldots, D_{N}\right]^{T},
\end{gathered}
$$

$I_{N}$ denotes the identity matrix. The parameters $K_{i}$ and $W_{i}$ are estimated through a MM approach. Adding or removing elementary models (5)-(6) is equivalent to an order change of the augmented model (10)-(11). 


\section{HYSTERESIS FUNCTION IDENTIFICATION AND REGIMES DETECTION WITH IMM ESTIMATOR}

The MM estimation [6] is based on a grid of the unknown parameters and variables. In the considered problem the unknown parameters are $K_{i}$ and the related with them variables $\Delta_{i}$. A set of grid values for $\Delta_{i}$, $i=1,2, \ldots, N$ is formed from physical restrictions, i.e. the maximal $\Delta_{i}$ is determined by the maximal amplitude of the input signal. The uncertainty interval of the spring constants, $\left[K_{i, \min }, K_{i, \max }\right]$ can be determined by looking to the minimal and maximal slopes of the measured hysteresis function. Then with each $\Delta_{i}$ a grid of spring constants $K_{i}$ (i.e. $K_{i, 1}, K_{i, 2}, \cdots, K_{i, q}$ ) is formed, covering well the interval $\left[K_{i, \min }, K_{i, \max }\right]$.

For each elementary model $i$ a separate IMM estimator is run, with $j=1,2, \ldots, q$ linear Kalman filters (KF)

$$
\begin{aligned}
& \hat{x}_{i, j}(k+1 / k)=F_{i, j} \hat{x}_{i, j}(k / k)+G_{i, j} u(k), \\
& \hat{x}_{i, j}(k+1 / k+1)=\hat{x}_{i, j}(k+1 / k)+K_{F_{i, j}}(k+1) v_{i, j}(k+1), \\
& v_{i, j}(k+1)=y(k+1)-\hat{y}_{i, j}(k+1 / k), \\
& P_{i, j}(k+1 / k)=F_{i, j} P_{i, j}(k / k) F_{i, j}^{T}+G_{\eta_{i, j}} Q_{i, j} G_{\eta_{i, j}}^{T}, \\
& S_{i, j}(k+1)=C_{i, j} P_{i, j}(k+1 / k) C_{i, j}^{T}+R_{i, j}, \\
& K_{F_{i, j}}(k+1)=P_{i, j}(k+1 / k) C_{i, j}^{T} S_{i, j}^{-1}(k+1), \\
& P_{i, j}(k+1 / k+1)=P_{i, j}(k+1 / k)- \\
& K_{F i, j}(k+1) S_{i, j}(k+1) K_{F_{i, j}}^{T}(k+1)
\end{aligned}
$$

working in parallel. Each Kalman filter uses the statespace model (5)-(6) with one of its three cases, depending on the switching parameter $f_{i, j}(k)$. The filtered and predicted estimates of $x_{i, j}(k)$ are $\hat{x}_{i, j}(k+1 / k+1)$ and $\hat{x}_{i, j}(k+1 / k)$ respectively; $v_{i, j}(k+1)$ and $S_{i, j}(k+1)$ are the innovation process and its covariance; $K_{F i, j}(k)$ is the Kalman filter gain (note the difference with $K_{i, j}$ which is the spring constant); $P_{i, j}(k)$ is the error covariance; $\hat{y}_{i, j}(k+1 / k)$ is the predicted output (according to eq. (6), with parameters of the form (7), (8) or (9), depending on the value of $\left.f_{i, j}(k)\right)$. The number of grid values for $\Delta_{i}$ is equal to the number $N$ of elementary models and the dimension of the state vector $\tilde{x}(k)$. The $q$ grid values for the spring constants determine the number of the KFs.

Using the MM approach [6], the estimated state $\hat{x}_{i}(k / k)$ of each $i$-th elementary model can be represented as a fusion of $j$ state estimates $\hat{x}_{i, j}(k / k)$ of the KFs , weighted by the mode probabilities $\mu_{i, j}(k)$

$$
\hat{x}_{i}(k / k)=\sum_{j=1}^{q} \hat{x}_{i, j}(k / k) \mu_{i, j}(k) .
$$

For each $i$-th elementary model an IMM filter is synthesized and its mode probabilities are used to compute the averaged elementary model state estimate. Each $i$-th estimate $\hat{K}_{i}(k)$ can be found as a fusion of values from the preliminary given set, probabilistically weighted by the IMM mode probabilities

$$
\hat{K}_{i}(k)=\sum_{j=1}^{q} K_{i, j} \mu_{i, j}(k) .
$$

Based on the grid values for $\Delta_{i}$ and the estimates $\hat{K}_{i}(k)$, the respective estimated force coefficients $\hat{W}_{i}(k)$ can be computed. The total estimated output (the hysteresis force) is the sum of estimated outputs from the elementary models

$$
\hat{F}_{h}(k / k)=\sum_{i=1}^{N} \hat{y}_{i}(k / k) \text {, }
$$

where

$$
\hat{y}_{i}(k / k)=\sum_{j=1}^{q} \hat{y}_{i, j}(k / k) \mu_{i, j}(k) .
$$

In the case of multiple elementary models, the innovation process $v_{i, j}(k+1)$ of the $j$-th Kalman filter within the $i$-th IMM estimator is computed as follows

$$
v_{i, j}(k+1)=y(k+1)-\hat{y}_{i, j}(k+1 / k)-\sum_{l=1, l \neq i}^{N} \hat{y}_{l}(k+1 / k),
$$

where the predicted outputs from the elementary models $l=1, \ldots, N, l \neq i$ are subtracted from the measured output $y(k+1)$. The output from each elementary model is computed as a fusion of the KFs' outputs weighted by the respective IMM mode probabilities.

For each elementary model the different behavior sticking or slipping can be determined by using the information provided by the probabilities. The probability whose value is greater than a preset threshold $\mu_{T}$, i.e.

$$
\max _{j} \mu_{i, j}(k)>\mu_{T}
$$

is considered as corresponding to the true regime.

\section{SiMULATION RESULTS}

\section{A. Results with one elementary model}

In the first simulation the hysteresis function consists of one elementary model (5)-(9) with $G_{\eta_{1, j}}=1$ and a known parameter $\Delta_{1}=0.55$. A hysteresis function of one elementary model corresponds to a backlash phenomenon. The parameters to identify are $K_{1}$ and $W_{1}$. A grid of values for $K_{1}$ is made, $K_{1, \text { grid }}=\{0.1,0.2,0.3,0.4\}$. The true coefficient $K_{1}=0.33$ is not on the grid, but it 
is within the uncertainty interval [0.1,0.4]. The transition probability matrix $\operatorname{Pr}$ and the initial mode probability vector $\mu(0)$ are

$$
\operatorname{Pr}=\left[\begin{array}{llll}
0.994 & 0.002 & 0.002 & 0.002 \\
0.002 & 0.994 & 0.002 & 0.002 \\
0.002 & 0.002 & 0.994 & 0.002 \\
0.002 & 0.002 & 0.002 & 0.994
\end{array}\right], \mu(0)=\left[\begin{array}{c}
1 / 4 \\
1 / 4 \\
1 / 4 \\
1 / 4
\end{array}\right] .
$$

The probabilities $\mu_{1, j}(0), j=1,2,3,4$, characterizing the possible values of $K_{1}$, are chosen to be equal. The initial true state variables are $x_{1, j}(0)=0$, whereas the initial estimated states $\hat{x}_{1, j}(0)$ are generated as random uniformly distributed numbers within the interval $(0,1)$. The Kalman filters are run with initial state estimate covariances $P_{1, j}=10^{5}$ and noise covariances: $Q_{1, j}=(0.001 N)^{2}, R_{1, j}=(0.1 \mu \mathrm{m})^{2}, Q_{u}=(0.05 \mu \mathrm{m})^{2}$. The input signal has been selected of the form [5]

$$
u(k)=\sin \left(\frac{2 \pi}{16} k\right) \sin \left(\frac{2 \pi * 30}{16} k\right)
$$

The data are obtained with a sampling interval $T=0.002 \mathrm{~s}$. The results presented are received by averaging over 30 Monte Carlo experiments. The real plant force $F_{h}(k)$, modeled by one element, the estimated plant force $\hat{F}_{h}(k)$, and the IMM probabilities are given in Figs. 4-6, respectively. The shape of the estimated hysteresis function is close to the shape of the modeled one as shown in Figs. 4 and 5. The mode probabilities

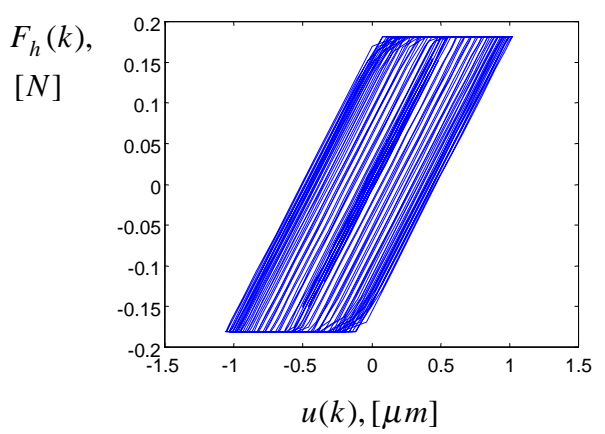

Fig. 4. Plant force modeled by one elementary model

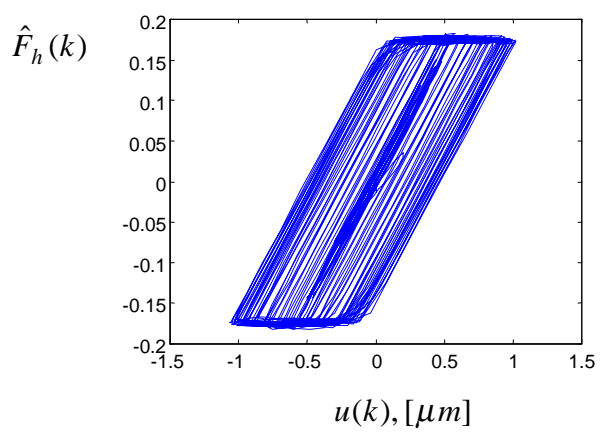

Fig. 5. Estimated plant force closest to the true parameter value (Fig. 6). The error $e(k)=F_{h}(k)-\hat{F}_{h}(k)$ between the estimated and real force is close to zero (Fig. 7). The estimation accuracy,

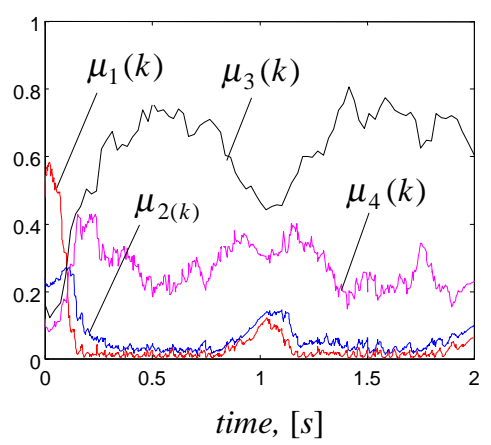

Fig. 6. The IMM mode probabilities

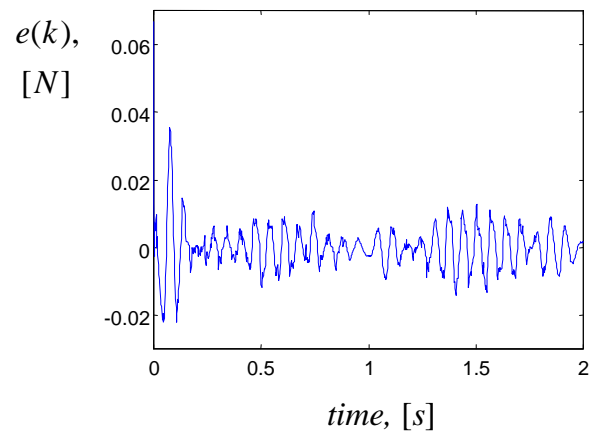

Fig. 7. Error between the estimated and real force

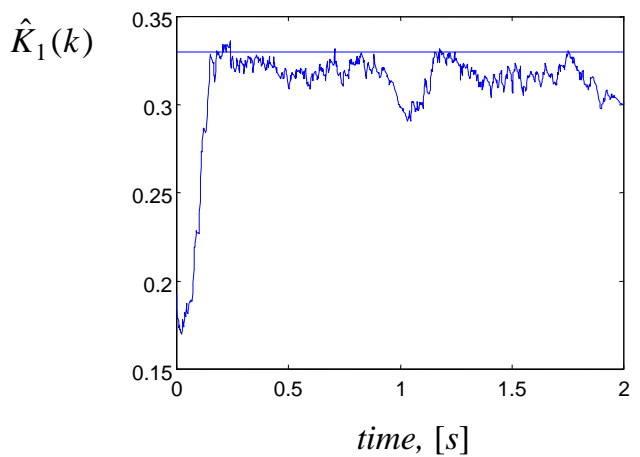

Fig. 8. The estimated coefficient $\hat{K}_{1}(k)$

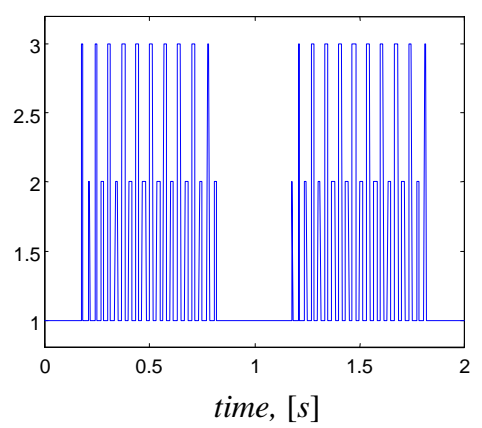

Fig. 9. Switching between the three "cases" in the real model for a single run 
characterized by this error with respect to the real force is less than $5 \%$. The estimated spring constant varies around its true value (Fig. 8). The three cases in the model (5)-(6) are denoted respectively by 1,2 and 3 . Switching between the three cases in the real model is given in Fig.9. The error between real switching and switching observed based on the biggest IMM probability (the third one) are presented in Fig. 10.

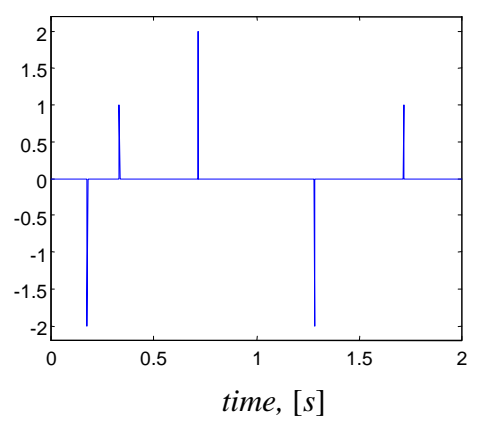

Fig. 10. Error between real switching and switching observed based on the biggest probability for a single run

\section{B. Results with more elementary models}

In this experiment the real hysteresis function is simulated with ten elementary models (5)-(9), with $G_{\eta_{i, j}}=1$ and

$\Delta_{i}=\{0.05,0.1,0.15,0.20,0.25,0.35,0.55,0.65,0.85,1\}$, $K_{i}=\{0.4,0.25,0.25,0.38,0.10,0.40,0.15,0.12,0.30,0.4\}$.

It means that the augmented model (10)-(11) is of order ten (unknown to the designer). The hysteresis behavior resulting from this augmented model is now approximated with a reduced order augmented model consisting of seven elementary models. Preliminary the uncertainty intervals for the parameters are determined, i.e. $\Delta_{i} \in(0,1]$ and $K_{i} \in[0.1,0.4]$.With each elementary model a separate IMM estimator is implemented with the same transition probability matrix, mode probabilities, initial conditions for the state estimates (random, uniformly distributed) and noise covariances as in the first experiment. The grid for $\Delta_{i}$ is : $\Delta_{i, \text { grid }}=\{0.03,0.1$, $0.2,0.4,0.6,0.8,1\}$ and for all spring constants: $K_{i, \text { grid }}$ $=\{0.1,0.2,0.3,0.4\}$. Compared to the exact values, three grid values of $\Delta_{i}$ coincide with their exact values, the others are not the same, but are within the uncertainty interval. Also some grid values of $K_{i}$ coincide with the exact ones, but the most of them - not. The real hysteresis force $F_{h}(k)$, its estimate $\hat{F}_{h}(k)$, the error $e(k)$ between them and the estimated coefficients $\hat{K}_{i}(k), i=1, \ldots, 7$ are given in Figs. 11-14. As seen from Figs. 11 and 12, the shapes of the estimated and real hysteresis functions are close. The estimation error is small (less than $5 \%$ ) (Fig.13). The estimated coefficients vary around their true values (Fig.14).

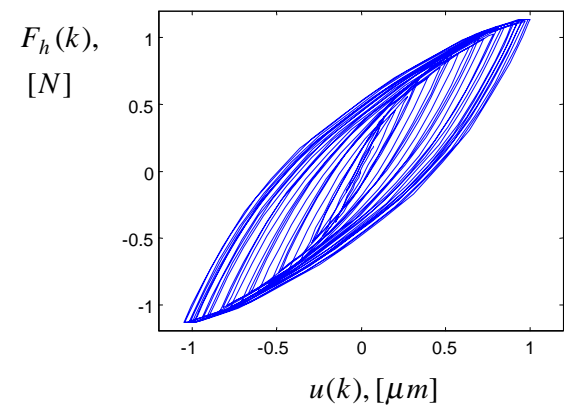

Fig. 11. The real hysteresis force, modeled by 10 elementary models

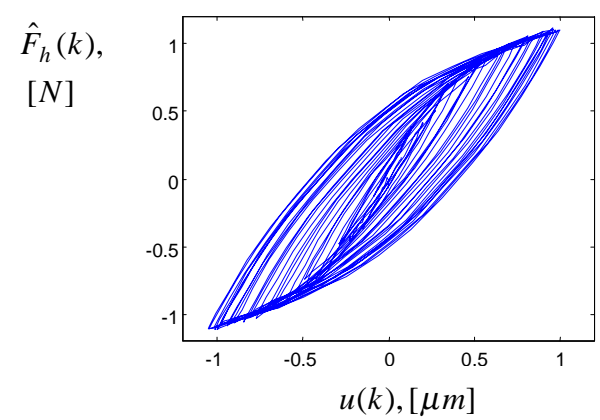

Fig. 12. The estimated hysteresis force by 7 elementary models

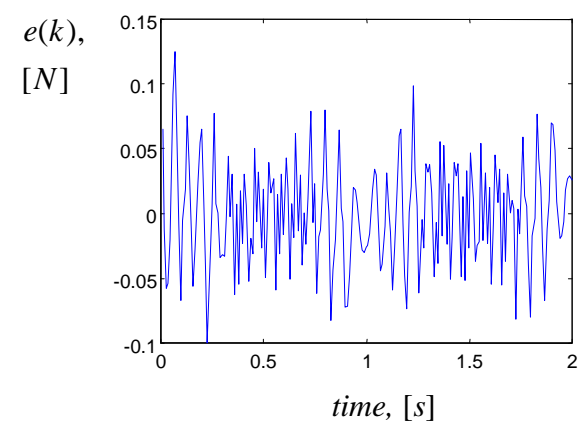

Fig. 13. Error between the estimated and real force

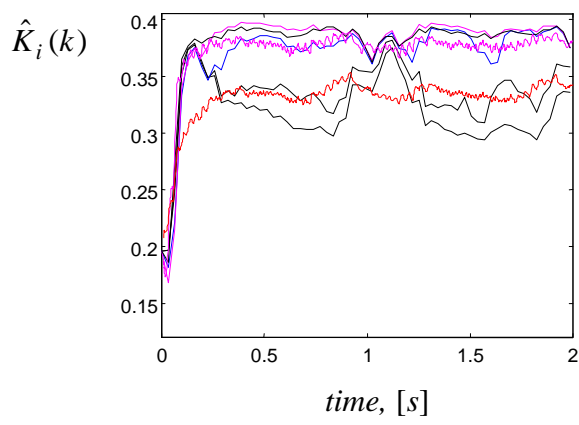

Fig. 14. Estimated coefficients $\hat{K}_{i}(k)$

The examples considered illustrate that through the MM approach the discontinuity (switching) in the state and output models can be overcome. 


\section{COMPARISON WITH THE RLSM}

Another possible solution for hysteresis function identification is developed in [5] using the recursive leastsquares method (RLSM). Results with high estimation accuracy are presented. The results obtained by the IMM estimator have a comparable accuracy of those from the RLSM. The RLSM requires an additional adaptation of the forgetting factor in the presence of changes in the model parameters, whereas the IMM approach possesses an inherent mechanism to reflect quickly the changes. The parallel structure of the model with three different cases and the IMM estimator give the possibility to model and identify hysteresis functions, characterized with a hard nonlinearity as switching. The IMM probabilities provide for each elementary model information about sticking and slipping behavior, that information is not available in the RLSM parameter estimates. In comparison with the results received by the RLSM [5], it is not possible to obtain negative values of the estimated parameters due to the fact that the probabilities can only have a positive sign. The IMM implementation does not require considerable computations. In every moment only one of the cases of the elementary model is active. For $N$ parallel elementary models and a grid with $q$ values for $K_{i}$, the number of active Kalman filters at each moment is $q N$. Advantage of the RLSM is that it works almost without initial information, whereas for the IMM the transition probability matrix should be preset. But the transition probabilities are chosen to correspond to the fact that one value of the spring coefficient is the most probable and this simplifies its determination. The crucial point of each MM estimator is the grid construction of the uncertain parameters. The true parameter values have to be within the preset uncertainty intervals.

\section{CONCLUSIONS}

This work presents a solution for static hysteresis function identification with a multiple model approach. The hysteresis function is described by a set of elementary models connected in parallel. This parallel structure of the model, in combination with the IMM estimator gives the possibility to reduce the nonlinear identification problem to a linear one. For the parameters (the spring constant and the force coefficient) of each elementary model, grids of possible values are preset taking into account physical restrictions. With each elementary model a separate IMM estimator is synthesized working by linear Kalman filters based on models with different parameters. The final estimate of each parameter represents a fusion of the values from the grid weighted by the IMM mode probabilities. The estimated output of each elementary model is a fusion of the weighted estimates of the Kalman filters by the probabilities, and the total hysteresis force represents the sum of the estimated outputs of all elementary models. A comparison with the recursive least-squares method is discussed.
Results from simulation experiments with one and more elementary models are presented.

\section{Acknowledgments}

H. Bruyninckx is a Postdoctoral Fellow of the Fund for Scientific Research-Flanders (F.W.O) in Belgium. Financial support by the Belgian Programme on InterUniversity Attraction Poles initiated by the Belgian State-Prime Minister's Office-Science Policy Programme (IUAP). The F.W.O. under grant G.0295.96N, K.U.Leuven's Concerted Research Action GOA/99/04 and grant I-808/98 with the Bulgarian National Science Fund are gratefully acknowledged.

\section{REFERENCES}

[1] Barabanov N., and R. Ortega, Necessary and Sufficient Condition for Passivity of the LuGre Friction Model, IEEE Trans. on Automatic Contol., Vol.45, No.4, pp.830-832, 2000.

[2] Canudas de Wit, C., H. Olsson, K. Åström and P. Lipchinsky, A New Model for Control of Systems with Friction, IEEE Trans. on Automatic Control, Vol. 40, No.5, pp.419-425, 1995.

[3] Goldfarb, M., and N. Celanovic, A Lumped Parameter Electromechanical Model for Describing the Nonlinear Behavior of Piezoelectric Actuators, Trans. of ASME, J. Dynamic Syst., Measurement, and Contr., Vol. 119, pp. 478-485, Sept 1997.

[4] Kuhnen, K. and Janocha H., Adaptive Inverse Control of Piezoelectric Actuators with Hysteresis Operators, Proc. of European Control Conf., 1999.

[5] Lampaert, V., and J. Swevers, On-line Identification of Hysteresis Functions with Nonlocal Memory, Proc. of Advanced Intelligent Mechatronics Conference, Como, Italy, 8-11 July, 2001.

[6] Li, X. R. Hybrid Estimation Techniques. Control and Dynamic Systems. (Ed. C.T.Leondes), Vol. 76, pp. 213-287, Academic Press, Inc., 1996.

[7] Mihaylova, L., State Estimation by IMM Filter in the Presence of Structural Uncertainty, Recent Advances in Signal Processing and Communications, Ed. N. Mastorakis, WSES Press, Greece, 1999, pp.83-88.

[8] Mihaylova, L., Semerdjiev, E., and X.R. Li, Detection and Localization of Faults in System Dynamics by IMM Estimator, Proc. of the Second Intern. Conf. on Multisource-Multisensor Information Fusion, 1999, Sunnyvale, California, USA, pp.937-943.

[9] Swevers, J. F. Al-Bender, C. Ganseman, T. Prajogo, An Integrated Friction Model Structure with Improved Presliding Behavior for Accurate Friction Compensation, IEEE Trans. on Automatic Control, Vol.45, No.4, pp.675-686, 2000.

[10] Zhang, Y., and X.R.Li, Detection and Diagnosis of Sensor and Actuator Failures Using IMM Estimator, IEEE Trans. on AES, Vol.34, No4, pp.1293-1312, 1998. 